\title{
Business process modelling: Comparing IDEF3 and CIMOSA
}

\author{
F.B Vernadat
}

Laboratoire de Génie Industriel et Production Mécanique

Université de Metz and ENIM

Ile du Saulcy, F-57012 Metz cedex 1, France

Tel: +33 (0)387346723 Fax: +33 (0)387346935

vernadat@poncelet.univ-metz.fr

\begin{abstract}
Enterprise Integration (EI) and business process reengineering (BPR) both require business process modelling methods to assess and reengineer enterprise behaviour and functionality in order to create better synergy among business entities. DEF3 and the CIMOSA process model are such methods. These two methods are reviewed and compared in this paper with regards to common business process modelling structures necessary to model industrial systems and manufacturing enterprises. Applications of the methods to the modelling of structured and semi-structured processes as well as to nondeterministic and concurrent processes are also discussed.
\end{abstract}

Keywords

Enterprise modelling, Enterprise Integration, BPR, Business processes, CIMOSA, IDEF3

\section{INTRODUCTION}

Enterprise Integration (EI) is concerned with braking down organisational barriers to improve synergetic effects and increase overall productivity within an enterprise or among cooperative enterprises (Bernus et al., 1996; Vernadat, 1996a). Central to EI is the modelling of enterprise activities and business processes to assess and rationalise enterprise functionality and enterprise behaviour (Vernadat, 1996b). CIMOSA (AMICE, 1993) and IDEF3 (Mayer et al., 1992) both promote a horizontal approach based on a modelling method enforcing the business process concept (as opposed to previous vertical approaches focusing on organisational functions). Both can be used in business process reengineering (BPR) or systems engineering projects. They are presented and compared in this paper as state of the art workflow methods to model manufacturing environments. 


\section{IDEF3}

IDEF3 is a process description capture method for Concurrent Engineering (CE), Total Quality Management (TQM) and BPR initiatives (Mayer et al., 1992). It has been developed as part of the IDEF suite of methods in the ICAM project supported by the US Air Force.

IDEF3 complements IDEF0, a functional modelling notation, and IDEF1x, an information system modelling technique based on the entity-relationship model. IDEF3 introduces a process-centred view to the IDEF method. It is itself complemented by DEF4, a method for modelling enterprise objects in the form of Object State Transition Networks (OSTNs) and IDEF5, a method for acquiring CIM ontologies (Benjamin et al., 1995).

Basic elements of the IDEF3 process description method include (Mayer et al., 1992):

- Units of Behaviour (UOBs) to define process steps (i.e. operations, activities or subprocesses)

- Three types of links (precedence, relational and object flow) to describe interrelations between two UOBs

- Three types of junctions (AND, XOR and OR junctions) which can be used as fan-in (convergent) and fan-out (divergent) junctions in either the synchronous or the asynchronous mode to formulate process flow description. This makes up to ten types of junction possibilities: four ANDs, four ORs and two XORs (the synchronous XOR has no meaning).

In addition, the concept of referent is used to refer to other models or previously defined parts of the model (e.g. a UOB, a junction, an object, a scenario, a note, an OSTN). Referents are also used to build "go-to" statements. Referents can be unconditional, asynchronous or synchronous.

Each of these constructs is defined in the IDEF3 formalism by means of a description template with predefined entries and by a graphical representation illustrated by Figure 1. Figures 2 and 3 provide examples of a process and one of its sub-processes expressed in IDEF3 about controlling items of an equipment to be assembled as a kit.

\section{CIMOSA}

CIMOSA is a reference architecture for enterprise integration in manufacturing developed by the ESPRIT Consortium AMICE (AMICE, 1993). It includes an enterprise modelling framework based on a process-centred approach, an enterprise engineering methodology based on the system life cycle concept and an integrating infrastructure for enterprise integration. In this paper, only its modelling framework, and especially its process model (Vernadat, 1993), is considered.

The CIMOSA process modelling language is a formal language and has no official graphical formalism because graphics are always ambiguous. It is made of a set of modelling constructs defined as object classes embedding declarative and procedural language statements. The language addresses the four CIMOSA views: function view, information 
view, resource view and organisation view. Thus, the set of constructs covers the concepts of domains, events, domain/business processes, enterprise activities, functional operations, functional entities (resources), enterprise objects, object views, organisation units and organisation cells (CIMOSA Association, 1996).

\section{UOB Box}

\begin{tabular}{|l|l|}
\hline \multicolumn{2}{|l|}{ UOB Label } \\
\hline Node \# & IDEF Ref\# \\
\hline
\end{tabular}

$$
\begin{aligned}
& \text { Function } \\
& \text { Activity } \\
& \text { Action }
\end{aligned}
$$

\section{Process \\ Operation \\ Event}

\section{Junction Boxes}

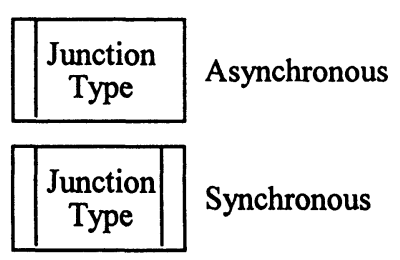

Junction Type

(Branch or Join):

AND (denoted \&)

OR (denoted 0 )

XOR (denoted X)

Links

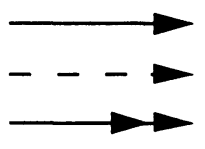

Precedence Link

Relational Link

Object Flow Link

\section{Referents}

\begin{tabular}{|c|}
\hline Referent Type/ID \\
\hline Locator \\
\hline
\end{tabular}

Unconditional

Referent

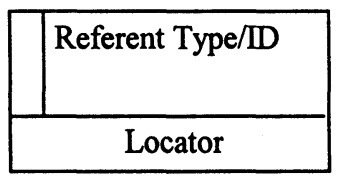

Asynchronous

Referent
Referent Types:

UOB

Elab

OSTN

Scenario

Artifact/Object Description

Note

Go-to

\begin{tabular}{|c|c|}
\hline & Referent Type/WD \\
\hline Locator \\
\hline
\end{tabular}

Sychronous

Referent

Figure 1 IDEF3 graphical formalism 


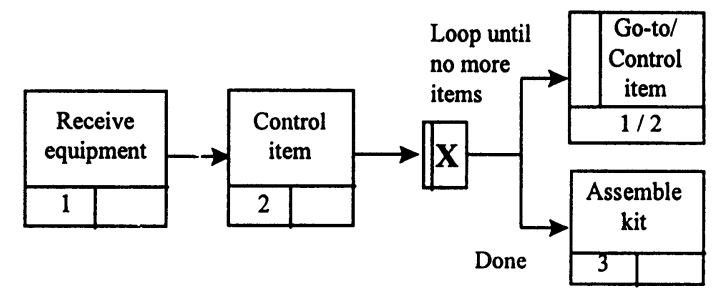

Figure 2 An IDEF3 process

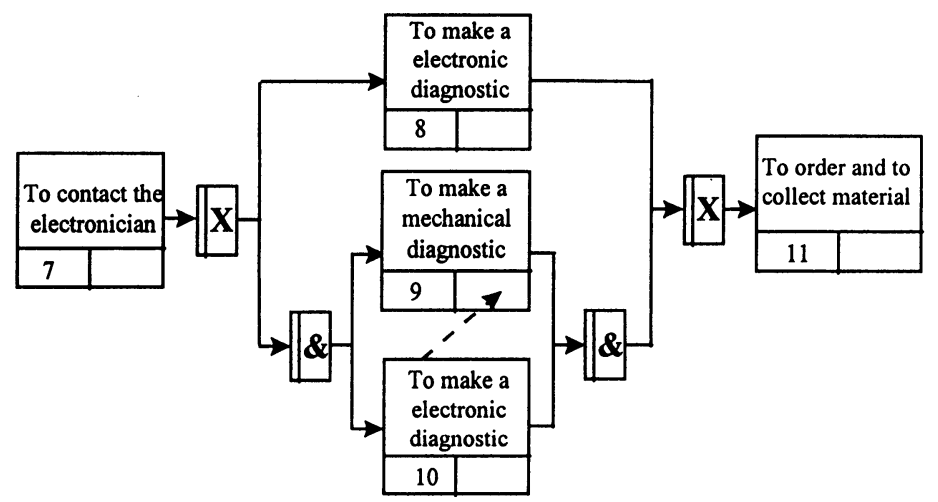

Figure 3 Control item sub-process

Activities, specifying the enterprise functionality, are defined by their name, their inputs (function, control and resource), their outputs (function, control, resource), the function transforming inputs into outputs and the set of all their possible termination statuses, called ending statuses. Function inputs and outputs are object states transformed by the activity, control inputs are objects used but not modified by the activity, control output gives the ending status of the activity upon its completion as well as events generated during the activity execution (if any), resource inputs are objects used as resources and resource outputs give information about the resource status at the end of the activity. Finally, the transformation function of an activity is in most cases defined by procedural statements involving functional operations, i.e. atomic commands (or requests) to be executed by functional entities (i.e. active resources or actors of the enterprise).

In CIMOSA, the control flow of a business process for describing enterprise behaviour is defined by means of a declarative language made of statements of the form:

\section{WHEN (condition) DO action}

called behavioural rules in which: 
- "condition" is a Boolean expression on events (or triggers) and/or expressions on termination statuses of process steps (denoted by ES(F) where ES stands for "ending status" and $\mathrm{F}$ is either an activity or a sub-process)

- "action" is the active part and indicates what to do next (i.e. it indicates the name(s) of activity(ies)/process(es) to execute when the triggering condition is satisfied).

\section{BUSINESS PROCESS MODELLING}

In this section, common workflow structures for modelling manufacturing environments or industrial systems and suitable for BPR are discussed on the basis of examples of Figures 2 and 3.

\subsection{Process start}

IDEF3 does not explicitly model process starts. In CIMOSA, domain processes are triggered by nothing but events (one event or a logical combination of event occurrences). Events indicate that a fact has happened requiring some action. The process start rule for the example of Figure 2 is as follows:

\section{WHEN (START WITH event_RE) DO Receive_Equipment}

indicating that an occurrence of Receive_Equipment will be executed as soon as an occurrence of event_RE occurs. If the process needs to be started by two events, event_1 and event_2, we can write:

\section{WHEN (START WITH event_1 AND event_2) DO Receive_Equipment}

\subsection{Sequential structures}

Both IDEF3 and CIMOSA provide a sequential structure. In IDEF3, this is modelled by the precedence link, an oriented arrow as shown in Figure 2. CIMOSA provides (1) a conditional sequential rule and (2) a forced sequential rule using the reserved word "any" meaning that whatever the ending status $\operatorname{ES}(x)$ of process step $x$ is, the process can go forward as soon as $x$ is completed. Examples for Figure 2 are:

$$
\begin{aligned}
& \text { WHEN (ES(Receive_Equipment) = any) DO Control_Item } \\
& \text { WHEN (ES(Control_item) = done) DO Assemble_Kit }
\end{aligned}
$$

\subsection{Conditional structures (branching)}

The conditional (or fork or branching) structure can be expressed either by a fan-out OR or by a fan-out XOR in IDEF3. The fan-out XOR indicates that one and only one alternative from several alternatives will be executed next (See, Figures 2 or 3). A fan-out OR indicates that one or several or even all of the possible alternatives can be executed next 
and in any order (i.e. $k$ among $n$ alternatives, with $1 \leq k \leq n$ ). In CIMOSA, only the XOR structure is provided. An example with two alternatives for Figure 2 is:

$$
\begin{aligned}
& \text { WHEN (ES(Control_item) = more-items) DO Control_Item } \\
& \text { WHEN (ES(Control_item) = done) DO Assemble_Kit }
\end{aligned}
$$

\subsection{Spawning structures}

The spawning structure is used either in synchronous or asynchronous mode to start $n$ parallel paths $(n \geq 2)$ in a workflow. In IDEF3, the fan-out AND junction box is used (Figure 3). In CIMOSA, the parallel operator (\&) is used in the action clause for asynchronous spawning as follows for the example of Figure 3 :

$$
\begin{aligned}
& \text { WHEN (ES(To_Contact_Electronician) }=\text { E\&Mdiag) DO } \\
& \text { To_Make_Mechanical_Diagnostic \& To_Make_Electronic_Diagnostic }
\end{aligned}
$$

For the synchronous mode, the reserved word SYNC must be used as follows:

$$
\begin{gathered}
\text { WHEN (ES(To_Contact_Electronician) }=\text { E\&Mdiag) DO } \\
\text { SYNC (To_Make_Mechanical_Diagnostic \& To_Make_Electronic_Diagnostic) }
\end{gathered}
$$

\subsection{Rendez-vous structures}

The rendez-vous structure is the symmetric structure of the spawning one. It is modelled by fan-in AND in IDEF3 and in CIMOSA by rules of the following form:

$$
\text { WHEN }(E S(E A i)=s i \text { AND ES(BPj)=sj AND ...) DO EAn) }
$$

For instance, for Figure 3 we can write:

$$
\begin{gathered}
\text { WHEN (ES(To_Make_Mechanical_Diagnostic) }=\text { MOK AND } \\
\text { ES(To_Make_Electronic_Diagnostic) }=\text { EOK) DO To_Order_And_Collect_Material }
\end{gathered}
$$

IDEF3 allows the use of synchronous fan-in AND. However, since all parallel paths being executed must be finished to complete a rendez-vous and proceed further, there is no real need for such a structure for discrete processes because control must wait until the completion of the latest path in the set. The structure can be useful for continuous processes.

\subsection{Loop structures}

Most workflow languages do not explicitly include a loop statement. Both in IDEF3 and CIMOSA, the loop must be constructed using a branching structure.

In IDEF3, a loop can be constructed in two ways. The first one is to use an XOR junction box followed by two branches: one going back to the loop entry point (an upstream 
UOB or junction box in the workflow) and the other one going to the rest of the workflow. The second way consists of using a go-to statement, i.e. using a referent from an XOR junction box onto a UOB or even a connector upstream in the workflow. IDEF3 recommends to use the second way because there is a rule in IDEF3 saying that it is not allowed to have arrows going from right to left in an IDEF3 diagram.

In CIMOSA, the loop is constructed using a branching rule as follows (assuming that EA-1 precedes EA-2 and EA-3 follows EA-2 in the workflow):

$$
\begin{gathered}
\text { WHEN }(E S(E A-2)=\text { s2) DO EA-3 } \\
\text { WHEN }(\text { ES }(E A-2)=\text { loop-condition) DO EA-1 }
\end{gathered}
$$

\subsection{Roll-back structures}

Roll-back structures can be found in engineering processes in which the process can execute up to a certain point where it is realised that it goes to a dead-end and that control must be passed to an earlier point upstream in the process to come back to a previous state and resume from that state, ignoring what was previously done after it. Neither IDEF3 nor CIMOSA supports this structure.

\subsection{Process end}

IDEF3 does not explicitly model process ends. In CIMOSA, the end of a process is specified by rules containing only the reserved word FINISH in the action part. For instance, for the sub-process of Figure 3, we will write:

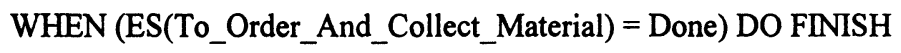

\subsection{Comparison}

To compare a workflow description in IDEF3 and CIMOSA, the full process behaviour of the sub-process of Figure 3 follows (for sub-processes, the starting rule has no event):

WHEN (START) DO To_Contact_Electronician

WHEN (ES(To_Contact_Electronician) = Ediag) DO To_Make_Electronic_Diagnostic

WHEN (ES(To_Contact_Electronician) = E\&Mdiag)

DO To_Make_Mechanical_Diagnostic \& To_Make_Electronic_Diagnostic

WHEN (ES(To_Make_Electronic_Diagnostic) = any)

DO To_Order_And_Collect_Material

WHEN (ES(To_Make_Mechanical_Diagnostic) $=$ MOK

AND ES(To_Make_Electronic_Diagnostic)=EOK)

DO To_Order_And_Collect_Material

WHEN $($ ES(To__Order_And_Collect_Material $)=$ Done $)$ DO FINISH 
As the example shows, the complete process behaviour can be expressed in CIMOSA in a formal and non ambiguous way using WHEN DO declarative statements. Because these statements can be expressed as Horn clauses or as Prolog statements, this makes possible the definition of consistency checking procedures based on logics.

\section{CONCURRENT PROCESSES}

Manufacturing enterprises can be viewed as complex dynamic systems made of many communicating and/or concurrent processes which need to be synchronised.

\subsection{Communicating processes}

There are two basic mechanisms to handle communicating processes (Hoare, 1985). Communication can be made:

- by message passing

- by shared memory

IDEF3 only provides the relational link (dashed arrow) between two UOBs (of the same process) or the use of a referent (from a UOB of a process onto a UOB of another process) to model interaction between two processes. The concept of messages does not exist in IDEF3.

In CIMOSA, communication between two processes can be established by message passing (through identified channels) or by shared memory at the activity level. This is realised by means of the use of predefined functional operations in the activity behaviour description. Available functional operations are (CIMOSA Association, 1996):

- request $(\mathrm{a}, \mathrm{c}, \mathrm{m})$ to ask for message or data $\mathrm{m}$ via channel $\mathrm{c}$ from activity a

- receive $(\mathrm{a}, \mathrm{c}, \mathrm{m})$ to receive message or data $\mathrm{m}$ via channel $\mathrm{c}$ from activity a

- $\operatorname{send}(\mathrm{a}, \mathrm{c}, \mathrm{m})$ to send message or data $\mathrm{m}$ via channel $\mathrm{c}$ to activity a ( $\mathrm{a}$ is undefined in the case of a broadcast)

- acknowledge (a) to let activity a know that the message/data sent has been received

The difficulty is to make sure that a given activity sends/receives the right message from the right receiver/sender activity (occurrence identification problem).

\subsection{Process synchronisation}

In many cases, concurrent processes need to be explicitly synchronised. They are implicitly synchronised in the model by resource access in the case of shared resources and by the flow of objects flowing from one process to another. However, other mechanisms influencing the control flow are required. IDEF3 only considers the flow of objects.

In CIMOSA, processes can be synchronised in three ways: 
- by events: Events are used to define starting conditions of processes. Events can be timers (clock times), real world happenings (e.g. customer order arrivals, machine break-downs) or requests. Events can come from the outside world (external requests) or from the system itself (raised by resources or by activities). In CIMOSA, an activity can raise an event (issued as a control output) by means of the CreateEvent predefined functional operation. Therefore, a process can generate an event which will start another process which can in turn generate results for the calling process using synchronisation by messages.

- by messages: An activity of a running process can send a message (a request) to another activity of another running process using primitives presented in section 5.1.This caters for synchronous or asynchronous communications (e.g. message passing or hand-shaking).

- by object flows: Objects created by one activity of a process are used by other activities of the same process or of other processes. Availability of object inputs is defined as pre-conditions to the start of activities.

\section{NON-DETERMISTIC PROCESSES}

\subsection{Non deterministic workflow}

Most business process modelling languages and tools are well suited to describe wellstructured processes, i.e. processes the control flow of which is completely known and deterministic. Few methods address ill-structured processes. CIMOSA provides two special behavioural rules to cope with these situations:

- The run-time choice rule: It is used in a process when there is an exclusive choice (XOR) among several alternatives at a given process step. Only one alternative will be executed as decided by the resource at run-time. Example:

$$
\text { WHEN }(E F(E F 1)=\text { s1) DO XOR }(E F 2, E F 3, E F 4)
$$

- The unordered set rule: It is used when a set of process steps, seen as a sub-process, must all be executed next by the same resource(s) but the exact order of execution is not known and will be decided by the resource(s) at run-time. Example ( $\mathrm{A}$ is the name of the set seen as a sub-process):

$$
\text { WHEN }(\mathrm{ES}(\mathrm{EF} 1)=\mathrm{s} 1) \mathrm{DO} \mathrm{A}=\mathrm{AND}(\mathrm{EF} 2, \mathrm{EF} 3, \mathrm{EF} 4)
$$

IDEF3 only provides the OR junction box (which is semantically ill-defined) to cope with these situations. Extensions to IDEF3 based on these rules and called IDEF3x to deal with semi-structured processes and non-structured activities have been recently proposed (El Mhamedi et al., 1996). 


\subsection{Exception handling}

Another way to deal with non-determinism is to address exceptions. Only foreseeable exceptions can be detected in the model. Other types of exceptions require the intervention of a control system or supervisor monitoring the execution of the model.

CIMOSA provides two mechanisms for exception handling both at the process and activity description levels, namely:

- time-out mechanism: the activity or the process is stopped after a fixed elapsed time, its ending status forced to a pre-defined value and control is forced according to that value,

- watch-dog mechanism: an exception condition is defined as a predicate and, when it happens to become true during activity or process execution, an event is created and generated which triggers an exception handling process. In this case, the faulty process is stopped and its ending status forced to a pre-defined value.

No such mechanisms are defined in IDEF3. Exceptions can only be documented and recorded as comments in plain text in the template form attached to UOBs.

\section{CONCLUSION AND RELEVANCE TO BPR}

In this paper, two methods for modelling business processes and enterprise activities have been compared on the basis of the most common structures necessary to model manufacturing environments like in EI or BPR projects. The first one, IDEF3, is a diagrammatic method well-suited as a business user language to capture system requirements. The second one, derived from CIMOSA, is more complete and can be applied at the business level for requirements definition but also at the design specification level as a semiformal description technique. Because of its graphical notation, IDEF3 has been found ambiguous in a number of situations which can be more properly handled by CIMOSA.

The paper has identified a number of typical situations that any workflow language should address for advanced business process modelling. The BPR analyst can therefore compare modelling languages of interest to him or her against these basic patterns and assess their suitability for his/her targeted BPR project.

\section{REFERENCES}

AMICE (1993) CIMOSA: Open System Architecture for CIM, $2^{\text {nd }}$ edn, Springer-Verlag, Berlin.

Benjamin, P.C., Menzel, C.P. Mayer, R.J. and Padmanaban, N. (1995) Toward a method for acquiring CIM ontologies. Int. J. of Computer-Integrated Manufacturing, 8(3): 225-234.

Bernus, P., Nemes, L. and Williams, T.J. (eds) (1996) Architectures for Enterprise Integration, Chapman \& Hall, London. 
CIMOSA Association (1996) CIMOSA Technical Baseline, Version 4.2. CIMOSA Association, Stockholmerstr. 7, D-71034 Böblingen, Germany, April.

El Mhamedi, A., Schroeder, V. and Vernadat, F. (1996) IDEF3x: A modelling and analysis method for semi-structured business processes and activities. Proc. Fourth Int. Conf. on Control, Automation, Robotics and Vision (ICARCV'96), Singapore, 3-6 December 1996.

Hoare, C.A.R. (1985) Communicating Sequential Processes. Prentice-Hall, Englewood Cliffs, NJ.

Mayer, R.J. et al. (1992) Information Integration for Concurrent Engineering (IICE), IDEF3 Process Description Capture Method Report, AL-TR-1992-0057, Air Force Systems Command, Wright-Patterson Air Force Base, Ohio, 45433.

Vernadat, F. (1993) CIMOSA: Enterprise modelling and integration using a processbased approach. In Information Infrastructure Systems for Manufacturing ( $\mathrm{H}$. Yoshikawa and J. Goossenaerts, eds.), North-Holland, Amsterdam. pp. 65-79.

Vernadat, F.B. (1996a) Enterprise Modeling and Integration: Principles and Applications, Chapman \& Hall, London.

Vernadat, F.B. (1996b). Enterprise Integration: On business process and enterprise activity modelling. Concurrent Engineering: Research and Applications, 4 (September): 219-228.

François Vernadat is a French and Canadian citizen. He holds a Master degree in Electronics and Automatic Control and obtained the Ph.D. degree in 1981 from University of Clermont, France. From 1981 till 1988, he has been a research officer at the Division of Electrical Engineering of the National Research Council of Canada, Ottawa. In 1988, he joined INRIA, a French research institute in computer science and automatic control. He is currently a professor at University of Metz, France.

His research interests include CIM, database technology and information systems, enterprise modelling, enterprise integration, manufacturing system specification, formal description techniques, Petri nets and workflow enactment. Beside his work on the $M^{*}$ methodology for CIM information systems, he has headed the development of a mainmemory database system (DBS/R) and was one of the chief architects of CIMOSA, an Open Systems Architecture for CIM initially developed as a series of ESPRIT projects (AMICE). He is the author of over 100 scientific papers in journals, conferences and books. He is the author of "Enterprise Modeling and Integration: Principles and Applications" (Chapman \& Hall) and a co-author of the book "Practice of Petri nets in Manufacturing" (Chapman \& Hall). He has been a co-chairman and invited speaker of several conferences on CIM. He is the European editor for the International Journal of Computer-Integrated Manufacturing and serves the scientific committee of other journals and conferences.

Dr. Vernadat served several times as a technical expert for the CIM programme of the Commission of the European Communities (CEC). He is the vice-chairman of the IFAC Technical Committee on Manufacturing Modelling, Management and Control and a member of the IFAC/IFIP Task Force on Architectures for Enterprise Integration. He is a member of the IEEE Computer Society, ACM, and SME. 